\title{
Menores e inseguridad. Analizando las "cifras" de la delincuencia juvenil en la ciudad de Rosario
}

\section{Daniela Polola}

Daniela Polola es Antropóloga, Becaria CONICET, Facultad de Humanidades y Artes, Universidad Nacional de Rosario.

Mail: danielapolola@ciudad.com.ar

\section{resumen}

En el presente trabajo realizaremos un acercamiento a la problemática de las personas de edad en conflicto con la ley penal en la ciudad de Rosario, Santa Fe, desarrollando, en una primera instancia, el estado de la cuestión en torno al tema, y describiendo la estructura burocrático-administrativa y judicial, junto con el marco legal que regula las intervenciones.

El objetivo central del trabajo radica en analizar la información estadística proporcionada por la Corte Suprema de Justicia sobre los Juzgados de Menores de la ciudad de Rosario, para analizar la situación de los menores de edad infractores a la ley, y así poder realizar una contrastación entre los datos oficiales y aquellos expuestos por la prensa gráfica, en particular en lo referente a temas de alta sensibilidad social tales como la (in)seguridad, los delitos cometidos por menores y los consecuentes pedidos de reducción de la edad de imputabilidad penal.

\section{palabras claves}

menores en conflicto con la ley penal / edad de imputabilidad / (in)seguridad

\section{summary}

In this paper we shall approach the issue of underage offenders in the city of Rosario, Santa Fe, developing the state of the art and describing the judicial and bureaucratic structure, including the legal frame that regulates intervention.

The main purpose of this paper is to analyze statistics produced by the Law Court about underage offenders in the city of Rosario to evaluate their situation so as to contrast official information with articles of the press, especially those referring to (in)security, juvenile crime and the consequent request of lowering the age of imputability amongst underage people.

\section{keywords}

underage offenders / age of imputability / (in)security 


\section{Introducción}

La problemática de las personas menores de edad en conflicto con la ley penal ha cobrado gran notoriedad en los últimos años en Argentina, en especial a través de los medios masivos de comunicación. Desde estos ámbitos se hace hincapié no sólo en el aumento de las causas cometidas por menores, sino también en los niveles de violencia que estos delitos involucran. Expresiones tales como la "ola delictiva", que "azota" a nuestras ciudades, se constituyen en moneda corriente en los artículos de la prensa gráfica y constantemente se enfatizan las demandas por parte de la ciudadanía de "mayor seguridad" al mismo tiempo que se apela, como una de las posibles soluciones, a la baja de la edad de imputabilidad de los menores.

Nuestro objetivo en este trabajo es realizar un análisis de la información estadística suministrada por la Corte Suprema de Justicia con respecto a causas cometidas por menores de edad en el período 1997-2006 en la ciudad de Rosario, provincia de Santa Fe, para efectuar una contrastación con los dichos de la prensa gráfica.

Como marco general del trabajo, se tomarán tanto los antecedentes de investigación como el contexto jurídico-burocrático que regula la intervención sobre menores de edad en nuestra provincia.

\section{Aproximación al estado del arte}

Desde la sanción, por parte de Naciones Unidas, de la Convención Internacional de los Derechos del Niño (CIDN) ${ }^{1}$, el tema niñez y adolescencia se convirtió en básico y prioritario en las agendas políticas de los diferentes países que la incorporaron -entre ellos Argentina- (al menos desde el punto de vista discursivo) y en función de esto, comenzó la proliferación de una gran cantidad de bibliografía en torno a la niñez, adolescencia y/o minoridad (dependiendo de la concepción doctrinaria de los investigadores y juristas) y sus derechos.

Existen numerosos antecedentes en torno a la problemática de la minoridad bajo procesos de intervención, ya sea del orden judicial como técnico-administrativo. Desde Donzelot (1990) se puede rastrear cómo se fue configurando en Francia, en torno a la infancia, una red de instituciones de control de las cuales la familia y la escuela son sólo el comienzo, culminando en las instituciones de reclusión, los juzgados de menores y las sociedades de beneficencia.

A nivel nacional, Dubaniewicz (1997) desarrolla un rastreo historiográfico de las instituciones de protección de menores desde la época colonial hasta 1997, describiendo cada una de las características principales de las instituciones de protección y castigo para menores abandonados o infractores.

Larrandart (1990), por su parte, realiza un racconto histórico a partir del cual el control institucionalizado (familia, escuela, policía) se tornó insuficiente debido a la gran ola inmigratoria que recibiera nuestro país a principios del siglo pasado y tal preocupación derivó en la creación de instituciones que pudieran ejercer dicho control, comenzando a tomar fuerza el discurso sobre la necesidad de "tutelar y proteger" a los menores y crear leyes e instituciones específicamente destinadas a ellos. Dentro de este contexto, de fuerte corte positivista e higienista, se sanciona, 
a nivel nacional, en 1919 la ley 10.903 de Patronato del Estado, que establecía la tutela del Estado tanto para los casos de comisión de un delito como para los de supuesto abandono de menores, enmarcada en la doctrina de la Situación Irregular ${ }^{2}$ (vigente en toda América Latina). El duro cuestionamiento que recibió la anómala situación legislativa generada por la vigencia de esta ley simultáneamente con la CIDN, provocó la proliferación de gran cantidad de bibliografía en torno al tema, fundamentalmente a cargo de juristas. García Méndez (2004) desarrolla un exhaustivo análisis de las leyes de menores en la Argentina y, comparativamente, en algunos países de América Latina que modificaron sus legislaciones en virtud de la incorporación de la CIDN. En una línea similar de trabajo, Beloff (2000) indaga en algunos aspectos negativos que implica la aplicación de la CIDN, y simultáneamente hace hincapié en la necesidad de atender a la "responsabilidad" de los jóvenes en conflicto con la ley penal, plasmado en un sistema de sanciones específicas que se base en medidas alternativas a la privación de la libertad, tales como la mediación y conciliación.

Desde las ciencias sociales, en los últimos quince años, el tema ha comenzado a adquirir interés y se han desarrollado investigaciones en el ámbito de la sociología y la antropología. Guemureman y Daroqui (2001) realizan un exhaustivo análisis acerca de las instituciones que surgen dentro del paradigma de la Situación Irregular y que se definen bajo el marco legislativo de la ley 10.903, conjuntamente con un amplio relevamiento de la situación normativa actual. Asimismo, describen el funcionamiento de una serie de instituciones que intervienen en menores, tales como los organismos técnico-administrativos, la policía y las comisarías de menores y cómo se incorporan al circuito judicial menores cuyos motivos de ingreso se vinculan a problemas de orden psiquiátrico o de droga-dependencia. Realizan una interesante contrastación entre el discurso de los jueces de menores y su práctica concreta -plasmada en la resolución de la situación que motivó la judicialización- a través de la sentencia.

Desde la antropología hay varios antecedentes, especialmente referidos a Buenos Aires. En cuanto al análisis de la estructura institucional de los juzgados de menores en la Ciudad de Buenos Aires, Villalta (1999) describe las peculiaridades de la justicia de menores y la problematización de los procesos de construcción de identidad en lo distintos agentes que la componen. Por su parte, Noriega (2002) realiza una indagación acerca de las "formas alternativas de resolución de conflictos" implementadas en el marco de la Red de Defensorías de Niños, Niñas y Adolescentes del gobierno de la Ciudad de Buenos Aires, tomando como contexto la Convención de los Derechos del Niño.

Grinberg (2004) focaliza en el análisis del entramado burocrático de protección y atención a la infancia en la Ciudad Autónoma de Buenos Aires, a partir de los cambios de orden político-administrativo, legislativo y judicial producidos luego de la sanción de la constitución local en 1996.

Miguez y González (2003), desde una perspectiva diferente, problematizan la noción clásica del sistema de minoridad como mecanismo de control social y enfatizan en la necesidad de un abordaje etnográfico en profundidad que permita 
desmitificar ciertos presupuestos. Sostienen que lejos de encontrarse en las instituciones de menores una estructura disciplinadora, lo que subyace es un "palimpsesto organizacional con prácticas contradictorias", que reproduce la violencia pero hacia el interior de los institutos, es decir, sobre los menores.

Kessler (2006), desde una perspectiva de corte etnográfico, se sumerge en las lógicas de "trabajo y provisión" entre las cuales pivotean jóvenes para los cuales el delito no es su único medio de ingresos, sino que también alternan con períodos de trabajo. Asimismo analiza las relaciones que se establecen dentro de los grupos de pares y la vinculación de los mismos con las drogas y el alcohol.

Desde el psicoanálisis, Degano (2004) problematiza la noción de "responsabilidad" en menores en conflicto con la ley penal a partir de una investigación realizada en Rosario. Cuestiona la rehabilitación como objetivo del tratamiento judicial. Elabora la categoría de "ficción jurídica" que define como la "característica ficcional del concepto de rehabilitación en el marco judicial de Menores" y finalmente propone una "política de la subjetividad" que involucre la responsabilización y la "producción de espacios para la subjetividad" de los menores.

Por otro lado, parecería ser una preocupación que se habría instalado en la agenda política como un área de vacancia y como consecuencia se han generado desde el Estado intentos -algo aislados y recientes- por activar una mirada más crítica sobre el tema, con actividades orientadas a la discusión de estas problemáticas, dirigidas tanto a los funcionarios de las instituciones de atención del área, como al público en general. ${ }^{3}$

Es interesante considerar también la producción en torno al tema generada desde los mismos organismos estatales como la Dirección Nacional de Política Criminal. ${ }^{4} \mathrm{Si}$ bien existen trabajos de relevamiento, en especial de tipo cuantitativo en cuanto a las "cifras" de la delincuencia juvenil, estos informes sólo se limitan a la exposición de los datos, sin que se vislumbre un interés por analizar, problematizar, de-construir esos datos, y convertirlos en información útil para sugerir líneas de acción en torno al tema. ${ }^{5}$

Desde el ámbito de las ciencias de la comunicación existe un significativo aporte a partir de investigaciones que abordan la incidencia de los medios masivos de comunicación en la conformación en la opinión pública de una imagen de "niñez delincuente y peligrosa" en virtud de un énfasis en las noticias policiales protagonizadas por menores de edad. Desde esta perspectiva, algunos trabajos indagan acerca de la construcción del problema de la violencia en los medios gráficos (Manguía, 2004).

Martini et al. (2002) analiza las crónicas policiales en los medios en torno a categorías de inclusión/exclusión y las representaciones del "nosotros" y "los otros" y cómo, a través de estas categorizaciones se naturalizan discursos hegemónicos, instalándose en la sociedad el tema de la inseguridad y la violencia por su constante aparición en los medios. El enemigo es el delincuente, en especial joven y pobre.

Vasilachis de Gialdino (2003), por su parte, evalúa el tratamiento que se realiza de los niños en situación de calle desde la prensa gráfica. En este sentido, analiza 
cómo a través de diversos procesos sintácticos y semánticos se enfatizan u ocultan los roles de estos niños, al mismo tiempo que se los estigmatiza por una situación (la de estar en la calle) que lejos de considerarse contingente se establece como inherente a ellos. Asimismo observa cómo dichos procesos construyen representaciones acerca de estos niños en la opinión pública que determinarán la manera en que serán vistos por la sociedad.

Calzado (2006) analiza, a través de las asambleas del Plan de Prevención del Delito de la ciudad de Buenos Aires, cómo éstas se convierten en generadoras de un discurso en torno a la inseguridad y al delito cometido por jóvenes, y esto ayuda a reforzar el binomio minoridad/inseguridad, para lo cual se conjugan tanto la opinión pública como los medios de comunicación. En consecuencia, esta construcción discursiva legitima respuestas políticas punitivas a problemas de orden estructural de nuestra sociedad.

Siguiendo esta idea del "miedo al delito" que se comienza a generalizar en la sociedad, Varela (2005) analiza la aparición de dicho concepto en la investigación criminológica británica y su utilización a nivel local como "sensación de inseguridad" asociado a la realización de la Encuesta de Victimización de la Dirección Nacional de Política Criminal, que establece el problema del delito antes de que los encuestados lo manifiesten como tal y en este sentido, también alude a cómo los medios de comunicación coadyuvan a instalar esta "sensación de inseguridad" en la población.

\section{Nuestro mapa institucional}

La Dirección Provincial del Menor, la Mujer y la Familia ${ }^{6}$ contaba inicialmente con el Programa de Menores en Conflicto con la Ley Penal, que comenzó a funcionar en abril de 1994. El 11 de agosto de 2000 este programa se convirtió en la Dirección del Menor en Conflicto con la Ley Penal que, en lugar de continuar dependiendo de la Secretaría de Estado de Promoción Comunitaria, pasó a estar bajo la órbita del Ministerio de Gobierno, Justicia y Culto.

En la actualidad, dichas instancias burocráticas están sufriendo una profunda reestructuración, paralelamente a una renovación de autoridades, a partir del cambio de gobierno de la provincia de Santa Fe a fines de 2007. En este contexto, se crea el Ministerio de Justicia y Derechos Humanos del cual pasa a depender la ya existente Subsecretaría de Asuntos Penales, que a su vez controla la Dirección Provincial del Menor en Conflicto con la Ley Penal (DPMCLP), organismo que rige para toda la provincia de Santa Fe.

La DPMCLP tiene incumbencia en toda la provincia de Santa Fe y se organiza en torno a tres grandes Programas dentro de los cuales se agrupan los más específicos. Estos son los Programas de Primera Intervención, por un lado Diagnóstico y Orientación, y Dispositivos de Asistencia en Seccionales Policiales. En segunda instancia los Programas de Tratamientos Alternativos a la privación de la libertad, que comprenden: Libertad Asistida, Centro de Día con Capacitación Laboral y emprendimientos productivos, y Centros de Asistencia de Victimas en Violencia Familiar y Delito Sexual, y por último, los Programas de Tratamiento con In- 


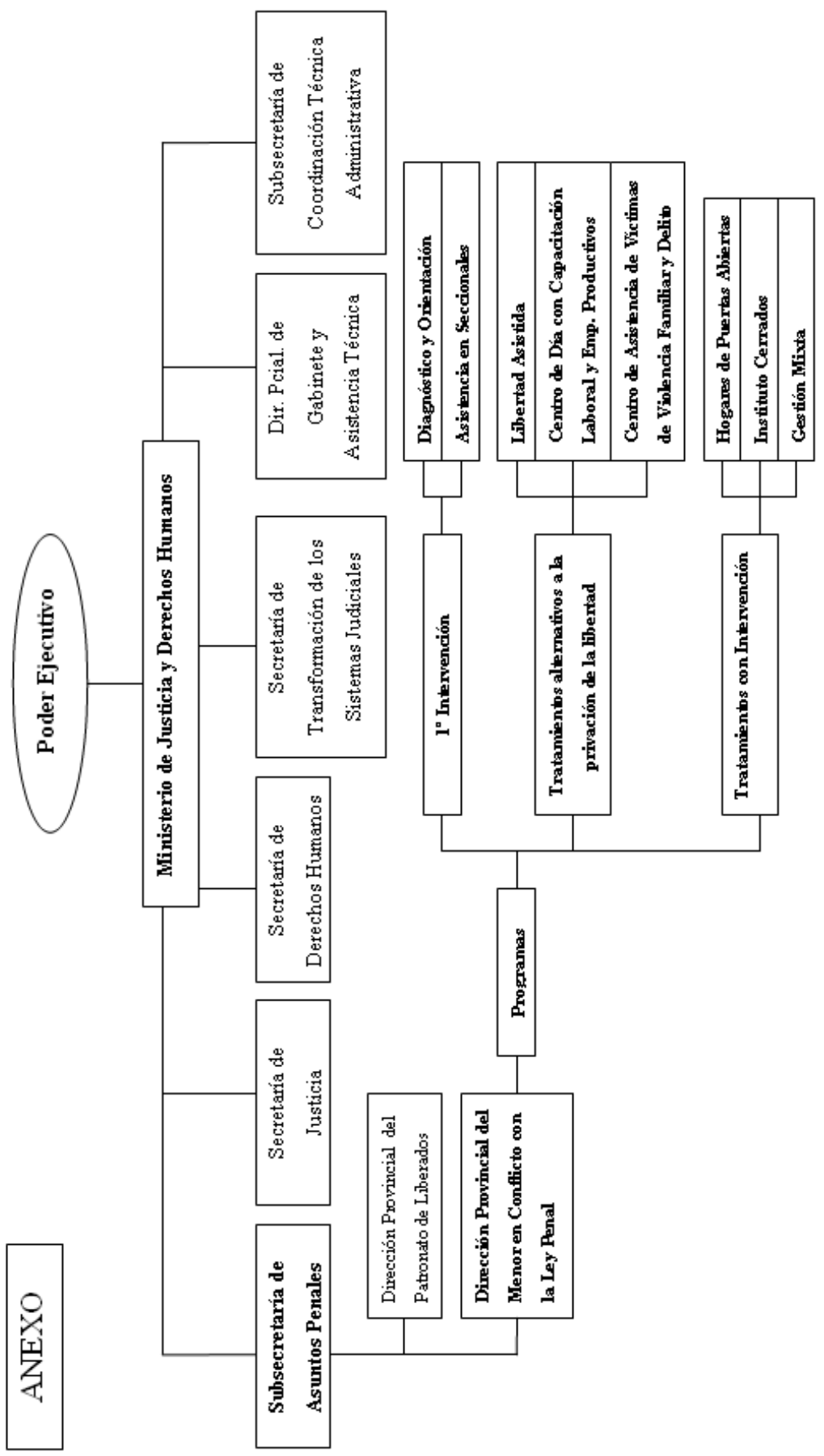


tervención, que pueden revestir tres modalidades: Hogares de Puertas Abiertas, Institutos Cerrados y Gestión Mixta.

Desde su creación a mediados de 2000 hasta fines de 2007, la DPMCLP estuvo bajo la órbita de control del Ministerio de Gobierno, Justicia y Culto, es decir, bajo la estructura de Seguridad, la misma instancia de la cual dependía tanto la Policía como el Servicio Penitenciario Provincial y todo el sistema de castigo vinculado a adultos. En este sentido, la decisión de que el ámbito penal en cuestiones referidas a menores (en lo que concierne a la provincia de Santa Fe), dependa de la misma estructura institucional de castigo de la cual dependen los mayores, implica que la justicia juvenil está concebida -al menos en parte- en los mismos términos que la justicia de adultos.

De todos modos, a la luz de los cambios institucionales que se están produciendo actualmente en los estamentos burocráticos provinciales, es muy aventurado adelantar una hipótesis sobre la morfología que adquirirán dichas instancias estatales y si, eventualmente, esos cambios redundarán en modificaciones en las prácticas de intervención sobre la población de menores en conflicto con la ley penal.

\section{Marco legal y situación jurídico-administrativa de los menores de edad en la ciudad de Rosario}

La ley nacional de fondo que regía desde 1919 en Argentina era la No 10.903 de Patronato de Menores que se correspondía con la doctrina de la Situación Irregular. Sin embargo, a partir de la aprobación de la CIDN por parte de la Asamblea General de las Naciones Unidas, el 20 de noviembre de 1989, su ratificación al año siguiente en nuestro país por Ley 23.849 del Congreso de la Nación y su posterior incorporación en 1994 a la Constitución Nacional, la situación jurídica de los menores de edad se tornó anómala dada la vigencia de dos leyes prácticamente antagónicas, durante casi quince años. Situación que sólo se modificó, en septiembre de 2005 al sancionarse la ley 26.061 de Protección Integral de los Derechos de las Niñas, Niños y Adolescentes, que automáticamente deroga la 10.903.

Si bien el texto de la nueva ley retoma en sus principios generales lo propugnado por la CIDN añade algunas cuestiones interesantes en las disposiciones generales en torno a las políticas públicas, en las cuales enfatiza el rol del Estado como garante del fortalecimiento familiar, la descentralización de los organismos de aplicación y de planes y programas, la gestión asociada de los organismos de gobierno en sus diferentes niveles coordinado con la sociedad civil, con una fiscalización permanente, y la promoción de redes intersectoriales locales. A pesar de que la sanción de esta ley constituye un avance importante desde el ámbito jurídico todavía se requieren adecuaciones, de tipo burocrático y de carácter procedimental, para lograr que ésta se efectivice plenamente.

En cuanto a la situación de la ciudad de la ciudad de Rosario, en 2001 su población era de 1.121.558 habitantes, según el Censo Nacional de Población, Hogares y Viviendas del mismo año. ${ }^{7}$ Para atender a los menores de edad, Rosario contaba con tres Juzgados de Menores, a los cuales se agregó un cuarto a mediados de 2006. Dichos juzgados, de acuerdo al marco legislativo que regula su práctica, 
intervenían tanto en el orden civil sobre menores en estado de abandono, como en el orden penal, sobre menores a los cuales se les imputaban hechos sancionados por la ley penal. ${ }^{8}$

A partir de la sanción de la ley 26.061 de Protección Integral de los Derechos de Niñas, Niños y Adolescentes gran parte de las competencias de los Juzgados de Menores pasan a los Juzgados de Familia, y a los órganos técnico-administrativos (Subsecretaría de la Niñez, Adolescencia y Familia) con lo cual, los Jueces de Menores restringen su área de incumbencia casi exclusivamente a lo relacionado con el ámbito penal.

\section{Las cifras de la delincuencia juvenil}

\subsection{El acceso a los datos}

Con este contexto jurídico-administrativo como marco general de nuestra investigación y, habiendo consultado la página web institucional del Poder Judicial de la Provincia de Santa Fe, constatamos que si bien existía información estadística correspondiente al fuero penal, ésta no figuraba desagregada entre mayores y menores de edad, motivo por el cual decidimos solicitar por nota material de tipo estadístico a la Corte Suprema de Justicia de la provincia de Santa Fe sobre Juzgados de Menores de la ciudad de Rosario, con respecto a: cantidad de causas por juzgados, cantidad de menores imputados, edad de los menores, tipo de delito, etc., del período 1997-2006. En base a nuestro conocimiento surgido de entrevistas informales con personal de un Juzgado de Menores, estábamos al tanto que existía un "Sistema de Gestión de Menores" informatizado en el cual se registraban este tipo de datos.

A partir de nuestro pedido concreto, se nos entregó un detalle estadístico desde el año 1997 al 2006. Algunos cuadros estadísticos adolecían de errores, que surgieron a partir de controles cruzados que efectuamos sobre los mismos (Rao, 1994). El origen de los errores puede deberse a varios motivos, entre ellos: un mal registro inicial de los datos, un incorrecto traspaso de los mismos del Sistema de Gestión de Menores a las planillas de tabulación de la Oficina de Estadísticas de la Corte o, inclusive, la falta de un rubro en el cual registrar datos, que incidiera en el total, pero no se viera reflejado en la desagregación.

El material se podía dividir en dos tipos: los datos ya tabulados y los datos "crudos", planillas directamente impresas del Sistema de Gestión de Menores sin procesamiento alguno. El análisis de ambos tipos de material implicó un arduo trabajo no solamente por los errores sino además, porque, los datos sobre delitos estaban ordenados alfabéticamente, sin ningún criterio específico, por lo cual decidimos clasificarlos según la tipificación de delitos establecida por el Código Penal de la Nación. Por otro lado, dentro de la categoría delitos aparecían ítems que no lo eran, por ejemplo figuras legales tales como el amparo.

\subsection{El análisis de los datos}

En la Tabla 1, nos interesa analizar la progresión de las causas iniciadas por Juzgado durante los diez últimos años. 
Tabla $N^{\circ}$ 1. Cantidad de causas ingresadas por Juzgado. Período 1997-2006

\begin{tabular}{|c|c|c|c|c|c|c|c|c|c|c|}
\hline Juzgado / Años & 1997 & 1998 & 1999 & 2000 & 2001 & 2002 & 2003 & 2004 & 2005 & 2006* \\
\hline Juzgado $N^{0} 1$ & 3368 & 2742 & 3146 & 3112 & 3022 & 3259 & 2915 & 2876 & 2645 & 1965 \\
\hline Juzgado $N^{\circ} 2$ & 2857 & 3251 & 3098 & 3200 & 3593 & 3657 & 3134 & 3208 & 4008 & 2855 \\
\hline Juzgado $\mathrm{N}^{0} 3$ & 3169 & 3158 & 3216 & 3494 & 3604 & 3524 & 3015 & 3001 & 2305 & 1889 \\
\hline Juzgado $N^{\circ} 4$ & .... & $\ldots$ & $\ldots$. & $\ldots$. & $\ldots$. & $\ldots$. & $\ldots$ & $\ldots$ & $\ldots$ & 1329 \\
\hline Total & 9394 & 9151 & 9460 & 9806 & 10219 & 10440 & 9064 & 9085 & 8958 & 8038 \\
\hline
\end{tabular}

* A mediados de este año se incorpora el Juzgado de Menores $\mathrm{N}^{\circ} 4$

Fuente: Elaboración propia en base a datos suministrados por la Corte Suprema de Justicia de la Provincia de Santa Fe.

\section{Gráfico $\mathbf{N}^{0}$ 1. Total de causas Ingresadas. Período 1997-2006}

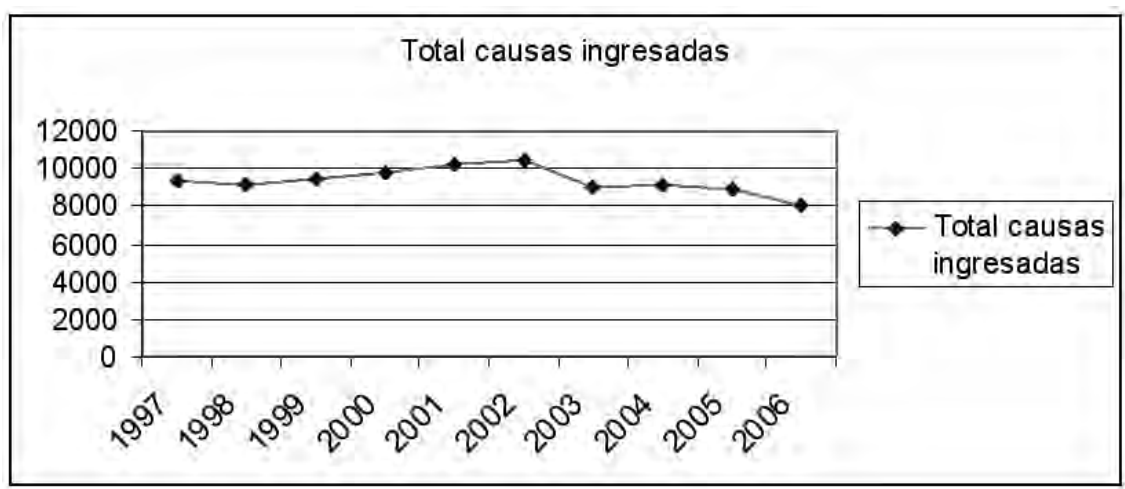

A partir de esta Tabla, podemos inferir que a pesar de las fluctuaciones, el número de causas ingresadas no muestra diferencias significativas, salvo en el período 2001-2002, lo cual podría ser atribuido a la crisis socio-política-económica que sufriera Argentina a fines del 2001. ${ }^{9}$

Según el Gráfico 1, los guarismos estarían dando cuenta que el fenómeno de la delincuencia juvenil -de acuerdo a las causas ingresadas en el fuero de Menoreslejos de estar in crescendo, y a pesar de algún pico puntual, se mantiene constante e incluso registra un leve descenso en los últimos años.

En la Tabla 2 se puede ver claramente que tanto las contravenciones de mayores contra menores ${ }^{10}$, como los recursos de Hábeas Corpus ${ }^{11}$, son los dos ítem que registran los guarismos más bajos, en relación con la averiguación de hechos/consultas, y fundamentalmente con respecto a los delitos o faltas cometidos por menores de edad que superan ampliamente a los demás ítem. No obstante esto, si realizamos un análisis en términos diacrónicos vemos que las contravenciones de mayores contra menores, la averiguación de hechos, y los recursos de 
Hábeas Corpus se mantienen relativamente constantes en el decenio analizado. En cambio, los delitos o faltas cometidas por menores registran una tendencia en aumento desde 1997 hasta 2002, año, este último, en que se verifica el máximo número de causas, y desde esa fecha comienza a descender bruscamente hasta el año 2003, registrando un ascenso en los años 2004 y 2005, que sin embargo nunca alcanza el pico de 2002, y que se encuentra en disminución en el último año.

\section{Tabla Nº 2. Desagregación de causas en Secretaría Penal}

\begin{tabular}{|l|r|r|r|r|r|r|r|r|r|r|}
\hline & 1997 & 1998 & 1999 & 2000 & 2001 & 2002 & 2003 & 2004 & 2005 & 2006 \\
\hline $\begin{array}{l}\text { Delitos o faltas cometidas } \\
\text { por menores }\end{array}$ & 7132 & 7465 & 7749 & 8361 & 8557 & 8915 & 5771 & 7202 & 7603 & 6948 \\
\hline $\begin{array}{l}\text { Contravenciones de } \\
\text { mayores c/menores }\end{array}$ & 4 & 0 & 1 & 4 & 204 & 0 & 0 & 192 & 0 & 0 \\
\hline $\begin{array}{l}\text { Averiguación de } \\
\text { hechos/denuncias/consult. }\end{array}$ & 413 & 253 & 476 & 255 & 254 & 325 & 248 & 264 & 233 & 167 \\
\hline $\begin{array}{l}\text { Recursos de Habeas Corpus } \\
\text { Total causas iniciadas }\end{array}$ & 76 & 9 & 19 & 14 & 12 & 29 & 26 & 19 & 17 & 15 \\
\hline Secretaría Penal & 7565 & 7727 & 8245 & 8634 & 9027 & 9269 & 6045 & 7677 & 7853 & 7130 \\
\hline
\end{tabular}

Fuente: Elaboración propia en base a datos suministrados por la Corte Suprema de Justicia de la Provincia de Santa Fe

\subsection{Menores de edad y tipos de delito cometidos. Un acercamiento desde el Código Penal}

En cuanto al tipo de delito cometido por menores de edad, establecimos un ordenamiento de acuerdo a la tipificación del Código Penal de la Nación, ya que en las planillas que nos suministrara la Corte Suprema los delitos estaban ordenados alfabéticamente, sin ningún otro criterio de clasificación, excepto la discriminación entre menores de hasta 16 años de edad, que la ley clasifica como no punibles y aquellos mayores de 16 años, que sí son pasibles de recibir sanción. ${ }^{12}$

Un dato importante es que en estas planillas de la Corte figura una leyenda que dice: "El cálculo de la edad tiene en cuenta la fecha de nacimiento y la fecha del hecho, si alguno de estos datos no posee información, no se contabiliza en la estadística". Esta aclaración cobra sentido si la relacionamos con el hecho de que los menores a veces mienten sobre su edad para evitar ser sancionados, entonces, si no se conocen los datos de nacimiento, o del hecho, no se lo consigna para evitar un registro erróneo. Esta situación implica que probablemente exista un subregistro de hechos, que nos impide realizar un control cruzado para establecer la confiabilidad de los mismos.

Tomamos un Juzgado de Menores como caso testigo para evaluar qué tipos de delitos poseen mayor incidencia. 
Tabla No ${ }^{\circ}$. Desagregación por tipo de delito. Período 2003-2006

\begin{tabular}{|c|c|c|c|}
\hline \multirow{3}{*}{ Tipo de delito } & \multicolumn{2}{|c|}{ Juzgado $\mathbf{N}^{0} 1$} & \\
\hline & $\begin{array}{c}\text { Menores no } \\
\text { punibles }\end{array}$ & $\begin{array}{l}\text { Menores } \\
\text { punibles }\end{array}$ & \\
\hline & (hasta 16 años) & (16 a 18 años) & Total \\
\hline contra las personas & 714 & 534 & 1248 \\
\hline 1. Homicidio & 30 & 30 & 60 \\
\hline 2. Tentativa de homicidio & 8 & 5 & 13 \\
\hline 3. Lesiones & 644 & 466 & 1110 \\
\hline 4. Abuso de armas & 30 & 32 & 62 \\
\hline 5. Agresión con armas & 2 & 0 & 2 \\
\hline 6. Aborto & 0 & 1 & 1 \\
\hline contra la integridad sexual & 45 & 22 & 67 \\
\hline 1. Abuso & 36 & 18 & 54 \\
\hline 2. Violación & 1 & 0 & 1 \\
\hline 3. Exhibiciones obscenas & 8 & 4 & 12 \\
\hline contra la libertad & 420 & 325 & 745 \\
\hline 1. Amenazas & 378 & 302 & 680 \\
\hline 2. Privación ilegal de la libertad & 2 & 5 & 7 \\
\hline 3. Violación de domicilio particular & 40 & 18 & 58 \\
\hline contra la propiedad & 3511 & 1915 & 5426 \\
\hline 1. Daño & 269 & 175 & 444 \\
\hline 2. Hurto & 716 & 355 & 1071 \\
\hline 3. Tentativa de hurto & 400 & 162 & 562 \\
\hline 4. Robo & 1298 & 1079 & 2377 \\
\hline 5. Tentativa de robo & 819 & 140 & 959 \\
\hline 6. Estafa y defraudación & 6 & 2 & 8 \\
\hline 7. Usurpación & 2 & 1 & 3 \\
\hline 8. Tentativa de extorsión & 1 & 1 & 2 \\
\hline contra la seguridad pública & 420 & 263 & 683 \\
\hline
\end{tabular}




\begin{tabular}{|l|r|r|r|}
\hline 1. Acopio de armas & 2 & 0 & 2 \\
\hline 2. Portación de arma de fuego & 28 & 32 & 60 \\
\hline 3. Tenencia de arma de uso civil & 1 & 2 & 3 \\
\hline 4. Incendios & 29 & 23 & 52 \\
\hline $\begin{array}{l}\text { 5. Infracción leyes policía sanitaria } \\
\text { animal }\end{array}$ & 360 & 206 & 566 \\
\hline contra la Adm. Pública & $\mathbf{9 7}$ & $\mathbf{1 3 7}$ & $\mathbf{2 3 4}$ \\
\hline 1. Encubrimiento & 31 & 45 & 76 \\
\hline 2. Falsa denuncia & 1 & 0 & 1 \\
\hline 3. Evasión & 17 & 17 & 34 \\
\hline 4. Desobediencia & 2 & 0 & 2 \\
\hline 5. Resistencia contra la autoridad & 46 & 72 & 118 \\
\hline 6. Atentado contra la autoridad & 0 & 1 & 1 \\
\hline 7. Falso testimonio & $\mathbf{5 2 2 4}$ & 2 & 2 \\
\hline Ley 23.737 (tenencia y tráfico & $\mathbf{1 7}$ & $\mathbf{2 7}$ & $\mathbf{4 4}$ \\
\hline estupefacientes) & & $\mathbf{3 2 2 3}$ & $\mathbf{8 4 4 7}$ \\
\hline Total & & & \\
\hline
\end{tabular}

Fuente: Elaboración propia en base a datos suministrados por la Corte Suprema de Justicia de la Provincia de Santa Fe

Con una simple visualización de la Tabla 3, podemos inferir que los delitos contra la propiedad son los que registran el mayor número de casos que corresponden a $64,24 \%$ del total. Y dentro de esta categoría los guarismos más altos se dan en robo y hurto, siendo el primero relativamente similar entre menores y mayores de 16 años. Sin embargo, en el hurto, los casos de menores de 16 años duplican a los mayores de esa edad. Dado que el robo implica la utilización de "fuerza en las cosas o (...) violencia física en las personas"13 , podríamos inferir que los menores de 16 años cometen hurtos más ocasionales y con menor grado de organización, sin la utilización de armas. Sin embargo si prestamos atención a la columna de delitos contra la seguridad pública vemos que tanto la tenencia de armas de guerra, como de uso civil, es muy similar en ambos estratos etarios con un leve aumento en los mayores de 16 años. Con lo cual los datos no confirmarían esta hipótesis.

En cuanto a los delitos contra la integridad sexual, el abuso es el que registra mayor incidencia, especialmente en menores de 16 años, que duplican a los cometidos por los mayores de 16 años.

Los delitos contra las personas constituyen el 14,78\% del total, pero dentro de los mismos, las lesiones ocupan el primer lugar, con $88,95 \%$, siendo los homici- 
dios sólo el 4,80\% del total de estos delitos.

A diferencia de las demás tipificaciones, los delitos contra la administración pública y los de tenencia y tráfico de estupefacientes, son los únicos que sufren incrementos en la franja etaria de 16 a 18 años de edad, que en el caso de los primeros, los mayores de 16 años representan el 58,54\% del total de estos delitos, mientras que con respeto a la ley de estupefacientes, sería el $61,36 \%$.

\section{Grafico No2: Desagregación por tipo de delito. Menores no punibles y puni- bles. Período 2003-2006}
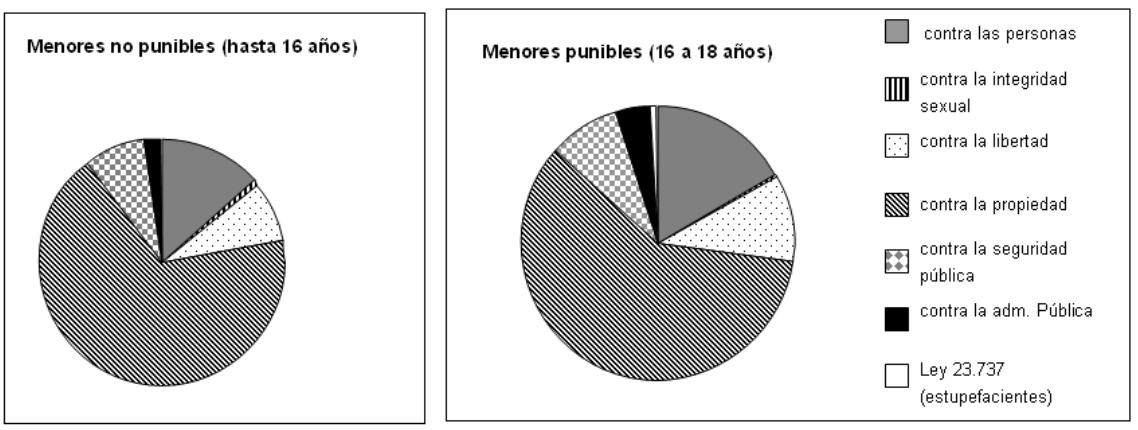

En otro orden de cosas, nos preguntamos por el objetivo que tiene el relevamiento de estos datos a nivel del fuero de menores, es decir, si efectivamente se utilizan en el mejoramiento del sistema, o si son aprovechados por otra instancia institucional para el diseño de políticas públicas para el sector. En otras palabras, si el dato se recopila con algún fin específico, o si sólo obedece a un requerimiento formal.

Los jueces del fuero penal consultados, sostienen que las estadísticas que producen los Juzgados sirven para su mismo control (Juez de Menores, Reg. $\mathrm{N}^{\circ} 2$, 2007) y que sería interesante que además puedan ser utilizadas para formular e implementar políticas hacia el sector e inclusive para modificar lo códigos de procedimientos (Juez de Cámara en lo Penal, Reg. N ${ }^{o}$ 1, 2007). De las entrevistas realizadas no surge que los datos, generados por los propios juzgados y procesados por la Oficina de Estadísticas de la Corte, se utilicen efectivamente para formular políticas públicas, para adaptar o mejorar cuestiones procedimentales o simplemente para realizar un diagnóstico de situación. De ser así, los jueces entrevistados no están al tanto del fin con que se recopilan dichos datos.

\section{La prensa gráfica y la inseguridad}

\subsection{Estableciendo lineamientos generales}

Es interesante analizar la información precedente a la luz de las noticias periodísticas que hacen hincapié en un aumento de los delitos cometidos por menores de edad dentro de una presunta "ola delictiva" que "azota" a nuestro país y especialmente a las ciudades más populosas. 
Para ello, se seleccionaron noticias periodísticas relacionadas con la delincuencia juvenil, pero especialmente intentando analizar si se plasma desde los funcionarios del área una preocupación por el tema. Escogimos el diario La Capital de la ciudad de Rosario (por ser el periódico con mayor tirada a nivel local) sobre el cual relevamos las notas del primer mes de cada cuatrimestre del período 2001-2006. ${ }^{14}$

A través del establecimiento de ejes a partir de los cuales ordenar las noticias periodísticas por año, pudimos constatar cómo el tema de la inseguridad atraviesa transversalmente los seis años analizados, siendo un común denominador en todo el país -según los medios- en un contexto marcado por la crisis de fines de 2001 en Argentina. También comienza a destacarse una notoria participación pública - especialmente la aparición de la clase media- en manifestaciones -a partir de las restrictivas medidas económicas implementadas por el entonces ministro de Economía Domingo Cavallo, en noviembre de 2001- y conjuntamente, comienzan a proliferar, a partir de 2002, las marchas solicitando mayor "seguridad".

Otro tema que aparece es el de la violencia institucional, es decir, muertes en cárceles o casos de "gatillo fácil". ${ }^{15}$ Y como contrapartida surge la preocupación por sanear la imagen de las fuerzas de seguridad, probablemente vinculado con lo anterior, pero también debido al alto índice de corrupción dentro de las mismas.

En este sentido, el tratamiento que realiza la prensa gráfica sobre el tema de la "(in)seguridad", sin duda contribuye a generar aquello que Hall define como "pánico moral", una reacción oficial desproporcionada en relación con aquello que se percibe como amenaza (Isla, 2003; Miguez y González, 2003). Y esto conduce a la demanda, por parte de la población, de más policía y mayor seguridad -otro de los ejes que aparece en el material periodístico- .

Ante esta situación, se postulan como posibles soluciones el establecimiento de planes de seguridad a nivel provincial; la construcción de más cárceles o la adaptación de edificios desocupados a tal fin. Como contracara, Apropo ${ }^{16}$ sostiene que los planes de seguridad provincial sólo implican dotar de más vehículos y mayor cantidad de efectivos policiales y que esto, aparte de no constituir una solución tiende a "la destrucción paulatina de la seguridad pública" (25/05/04).

En otros casos, la visibilidad que la prensa le otorga a ciertos sucesos contribuye a denunciar situaciones problemáticas. Por ejemplo, a partir de algunos motines protagonizados por menores de edad alojados en comisarías, se resuelve que sean trasladados a institutos de internación dependientes de la DPMCLP, ya sea de régimen abierto o cerrado, para solucionar una situación que de hecho ya era irregular: que existan personas menores de edad detenidas en comisarías.

Por otro lado, las políticas públicas destinadas a menores de edad que estén o hayan estado en conflicto con la ley penal son muy escasas, sólo en dos notas (de un total de noventa y una relevadas) se menciona un programa de deporte, alfabetización, educación formal y talleres de oficios gestionado a través de la DPMCLP $(29 / 09 / 02)$ y, en otra, la propuesta de fomentar actividades deportivas como factor de contención social (09/05/05).

Un Juez de Menores se refiere a esta situación de la siguiente manera: "Para 
extraerlo del delito, es decir, un ejemplo tonto, tonto: no puede haber ningún pibe que haya cometido un delito aunque sea el más grave, que se vaya en libertad y no tenga, por ejemplo, trabajo. O no tenga, que no termine sus estudios..." (Juez de Menores: 2007).

Asimismo, la consulta a especialistas en el tema-cuando se realiza-se ve restringida a abogados, que participan en instituciones del Estado, o a criminólogos. Algo notorio es el casi nulo recurso de este medio gráfico a incluir la opinión de cientistas sociales, ya que en los seis años relevados sólo aparece una nota en la cual opina sobre el tema una socióloga.

Al respecto, en un informe publicado por la ONG Periodismo Sociall ${ }^{17}$, la Dra. Trumper sostiene que “...es problema de las sociedades científicas, que no sabemos llegar o creemos que no nos van a tener en cuenta. Desconocemos el trabajo de los periodistas. No hay relación fuera de la coyuntura noticiosa... Tendríamos que usar más los medios de prensa para que pueda llegar más información veraz a la prensa" (Periodismo Social, 2006:20).

La duda que surge entonces es: ¿la prensa se aleja de los cientistas sociales o los cientistas sociales no se acercan a la prensa? ${ }^{18}$

\subsection{Los menores infractores y la prensa gráfica}

Es interesante, entonces, analizar ahora las cifras de la delincuencia juvenil en cruce con los dichos de la prensa. Para ello tomaremos una serie de noticias, que consideramos paradigmáticas en la construcción de la "inseguridad" vinculada a delitos cometidos por menores de edad.

Un titular anuncia "A contrapelo del país, en la provincia no disminuye el índice de delitos" (30/05/2005) y más adelante expresa: "Mientras la media nacional indica una merma de 2,12\% entre los años 2003 y 2004, en Santa Fe se registró un incremento del 4,29\%". Si bien no queda claro si estos porcentajes incluyen a los delitos cometidos por menores de edad, lo más probable es que sí los tengan en cuenta ${ }^{19}$, entonces, parecería ser que nuestros datos sobre menores siguen la tendencia enunciada para el resto del país (con una disminución en 2003-2004), que sin embargo, no coincidiría con la esbozada por el medio gráfico para la provincia de Santa Fe.

"El delito en menores sube con el colapso del sistema tutelar" (18/05/2005) y en el subtítulo continúa "En un año calendario en Rosario se abrieron más de 6 mil causas", lo cual, según nuestros informes de hecho es cierto, porque en 2005 la cantidad de causas fue 8958. No obstante, este guarismo está por debajo de los nueve años anteriores, lo cual implica el riesgo que existe al realizar aseveraciones de este tipo en un medio masivo de comunicación sin contextualizar los datos con los de años previos, permitiendo que el lector pueda sacar sus propias conclusiones.

Es notorio que a pesar de la resonancia de la cual goza el tema de menores en conflicto con la ley penal, no se encuentren datos oficiales de fácil acceso sobre las cifras de este fenómeno. En este sentido, Daroqui y Guemureman señalan que: “...la ausencia de producción de información sobre un tema candente como la delincuencia juvenil, permite, en contraposición, la vulneración de derechos, ya que 
promueve -por omisión- la posibilidad de decir virtualmente "cualquier cosa" sobre los adolescentes y jóvenes y el diseño responsable de políticas públicas sobre la desinformación" (citado en Guemureman, 2004:48).

En otra noticia se hace hincapié en los delitos cometidos en un turno judicial, que equivale a un mes: "En un mes, hubo más de 600 delitos de menores en el área de Rosario" (12/05/2003). Si calculamos la media de las causas iniciadas en el período 1997-2006 obtendríamos 9300 por año, y tendríamos alrededor de 775 causas por mes, lo cual supera al número mencionado en esta nota, pero que, volvemos a remarcar, tomado el dato aisladamente, sin una correcta contextualización, puede resultar "alto" para el lector, pero ¿alto con respecto a qué?

Martín et al. (2002:88) señala: "Se asume que la agenda policial de los medios excluye a la ciudadanía de la posibilidad de participación activa en el debate público por la reiteración de representaciones simplificadas y reduccionistas de la realidad construidas por los medios y por el modo incompleto en que se publican las noticias sobre el crimen."

Estos discursos contribuyen a construir una imagen dicotomizada de "nosotros" y "los otros" (Calzado, 2006; Martín et al., 2002) que refuerza los estereotipos y asocia joven con delincuente, al mismo tiempo que ubica los actos delictivos fuera de todo contexto social y como simple resultado de la voluntad individual de los sujetos.

En este sentido, no debemos olvidar que la prensa construye discursos que se constituyen en verdad y son apenas cuestionados o tomados críticamente por la opinión pública: "La legitimidad que tiene históricamente la prensa le concede el estatuto de voz autorizada, y que se deriva de su coincidencia con el discurso hegemónico, "oficial", es decir con las fuentes que producen autoridad." (Martín et al., 2002:90 - subrayado original)

Entonces, noticias que mencionan temas tales como "La ola de inseguridad que padece Rosario desató un fuego cruzado entre la Municipalidad y la Casa Gris" (30/05/2003 - el subrayado es nuestro) desatan polémica en la opinión pública y llevan a que la solución se encauce por la vía de la reducción de la edad de imputabilidad de los menores, tal y como se manifiesta en este titular: "El gobierno analiza bajar a 14 años la edad para imputar delitos. El funcionario negó que se trate de una reacción al reclamo del empresario Juan Carlos Blumberg" $(18 / 05 / 2005) .^{20}$

Según Calzado (2006:28) "este tipo de estados de conmoción social no necesariamente coinciden con mayores índices delictivos". En este sentido, en un artículo publicado el 19/09/04 cuyo titular era: "Rosario: la mayoría de los crímenes son por conflictos personales y no por robos", se hace hincapié en que "...una caída del número de crímenes en Rosario, reflejaría un declive del homicidio como emergente de la inseguridad pública" y a continuación agrega: "Sin embargo, lejos de decaer, el temor público a la delincuencia aumenta". En el mismo artículo, el criminólogo Máximo Sozzo sostiene que los delitos contra las personas -puntualmente los homicidios- no se hayan en la base del temor público sino, por el contrario, los que atentan contra la propiedad.

Un informe presentado por la ONG Periodismo Social menciona que: "La Vio- 
lencia, en sus diversas formas, ha sido el tema más tratado por el conjunto de los diarios nacionales argentinos durante 2004 en asuntos de niñez y adolescencia (26,1\% del total de notas)" (Informe Anual 2005, pp.22). Pero agrega que “...los textos sobre chicos víctimas de actos violentos quintuplican -incluido abuso y explotación sexual- a aquellos en los que fueron agentes: el 65,6\% de las piezas sobre violencia los tiene como víctimas, el 12,6\%, como agentes" (Ibídem). ${ }^{21}$

Por otro lado, debemos tener en cuenta que "el crimen vende" y que: "La información periodística sobre el "mundo del delito" constituye una de las más poderosas narrativas explicativas que ha diseñado lo modernidad y se ha instaurado...como forma más o menos sutil de control social" (Martín et al., 2002:87 - subrayado original).

Este cruce del material periodístico con los datos provenientes de la Corte Suprema nos indica lo cuidadosos que debemos ser al establecer conclusiones, para que éstas no impliquen una generalización incorrecta o una total descontextualización del problema en términos comparativos.

\section{Algunas reflexiones finales. Retomando ideas}

En el presente trabajo desarrollamos el complejo entramado jurídico-administrativo que interviene sobre menores de edad, a través de una somera descripción de las instituciones del área del Estado provincial santafesino, con incumbencia en la ciudad de Rosario. Asimismo realizamos un acercamiento al estado del arte para, finalmente, sumergirnos en el análisis de las estadísticas oficiales en contraste con el tratamiento de la problemática que se realiza desde la prensa gráfica.

Como vimos, la situación de la provincia es paradigmática ya que la estructura burocrática de la DPMCLP (desde su creación hasta fines del 2007) estuvo vinculada al Ministerio del cual dependía todo el sistema penal de adultos. En este sentido, la decisión política de incluir a los menores dentro de esta instancia institucional implicaba, de algún modo, que el sistema de justicia juvenil estaba siendo pensado en términos de castigo más que de reinserción de los jóvenes en ámbitos de contención social que les posibilitaran salir de la delincuencia. Con lo cual, se continuaba con un tratamiento tutelar de los menores que desatiende el interés en la responsabilización subjetiva de los mismos. Esta decisión en parte contradice el espíritu de los tratados y convenciones internacionales a los cuales adhirió Argentina. ${ }^{22}$

Sin embargo, debemos puntualizar también que, a la luz de las recientes reestructuraciones institucionales que está sufriendo la provincia de Santa Fe a partir del cambio de gobierno en 2007, es demasiado aventurado adelantar el curso que tomará el tratamiento de la problemática a nivel provincial. Situación que, probablemente, sólo podrá ser evaluada a largo plazo.

En cuanto a las "cifras" de la delincuencia juvenil, los datos aportados por la Corte Suprema reflejan no sólo una relativa estabilidad en el número de causas, sino inclusive, un leve descenso de las mismas en el último año. Sin embargo, desde los medios de comunicación se construyen imágenes en torno a la (in)seguridad que ubican a los menores de edad como uno de los principales causantes del pro- 
blema y en este sentido, la exacerbación de la sensibilidad social en torno al tema genera reacciones de la opinión pública que se orientan hacia el endurecimiento de las penas, la baja de la edad de imputabilidad penal y otras medidas de corte punitivo que, lejos de resolver el problema, son sólo medidas paliativas de muy corto alcance.

En este sentido, el problema no son los datos que maneja la prensa, sino la utilización que se realiza de ellos, especialmente cuando se los analiza descontextualizadamente, sin otorgar al lector elementos que le permitan evaluar críticamente la información.

En cuanto a las políticas públicas destinadas al sector, tanto desde lo analizado a partir de los medios masivos de comunicación como a través de las voces de los jueces del fuero penal, parece vislumbrarse una ausencia del tema en la agenda política, en especial en torno a programas que tiendan a la reinserción social de adolescentes infractores a la ley penal en ámbitos de contención, ya sean vinculados al estudio o al trabajo.

Finalmente creemos que no basta con sancionar leyes "supuestamente" aggiornadas al contexto internacional -que luego fallan en el momento de la implementación, porque no fueron realizadas las reestructuraciones institucionales suficientes para llevarlas a cabo- ni el agregado de Juzgados de Menores, ni medidas paliativas aisladas, sino que, una política juvenil comprometida con nuestra realidad social implica comprender la complejidad del problema cuya raigambre es eminentemente social y su abordaje debe ser también pensado en esos términos, articulando a nivel horizontal los diferentes Ministerios y a nivel transversal los estamentos nacional, provincial y municipal y, fundamentalmente, abordando el problema desde una perspectiva holística que entienda a la niñez y a la adolescencia no como parcialidades sino como una totalidad.

\section{Referencias}

1. El 20 de noviembre de 1989 es aprobada por la Asamblea General de las Naciones Unidas la Convención Internacional de los Derechos del Niño y ratificada en nuestro país por Ley 23.849 del Congreso de la Nación y en 1994 incorporada a la Constitución Nacional.

2. Para mayor información sobre la doctrina de la Situación Irregular y de la Protección Integral y las leyes que se inscriben dentro de cada una de ellas en la República Argentina, consúltese POLOLA, Daniela (2004) De Niños y de Menores. Los profesionales y el complejo mapa institucional de la "minoridad" en la ciudad de Rosario, Tesis de licenciatura, Escuela de Antropología, Facultad de Humanidades y Artes, Universidad Nacional del Rosario.

3. $\mathrm{I}^{\mathrm{o}}$ Seminario Internacional "Respuesta institucional a la niñez en riesgo" (marzo de 2005) y II ${ }^{\circ}$ Seminario Internacional "La violencia en la familia, escuela y sociedad" (noviembre de 2006), ambos organizados por la Defensoría del Pueblo de la Provincia de Santa Fe, $1^{\circ}$ Foro sobre Niñez, Adolescencia y Familia: El Sistema de Protección Integral de Derechos en el marco de la nueva Ley Nacional 26.061 (agosto de 2006), organizado por la Municipalidad de Rosario.

4. Cfr. Dirección Nacional de Política Criminal, Ministerio de Justicia y Derechos Humanos de la Nación. Adolescentes infractores a la ley penal. Tribunales Orales de Menores. 2002 Informe de la http://www.polcrim.jus.gov.ar; L. ELBERT; V. VASILE; F. REYES (2002), Situación normativa de las personas menores de 18 años imputadas de la comisión de un delito, Dirección Nacional de Política Criminal, Ministerio de Justicia y Derechos Humanos de la Nación. http://www.polcrim.jus.gov.ar; L. ELBERT; V. VASILE; F. REYES, Violencia juvenil y seguridad ciudadana. Problemas y soluciones, 
Informe de la Dirección Nacional de Política Criminal, Ministerio de Justicia y Derechos Humanos de la Nación. http://www.polcrim.jus.gov.ar

5. Guemureman (2004:3) señala que actualmente existen más "usinas generadoras de datos" que las que había hace algunos años, probablemente porque se visualiza como positiva la información. Al mismo tiempo señala que "Hoy por hoy existe muchísima más información disponible, pero por ese mismo motivo también hay mayor fragmentación y atomización. La lectura integrada resulta mucho más difícil...".

6. Esta Dirección recientemente cambió su nombre por el de Subsecretaría de Niñez, Adolescencia y Familia como parte de la adecuación a la ley 26.061 de Protección Integral de los Derechos de niñas, niños y adolescentes sancionada en octubre de 2005. Sin embargo, en el presente trabajo utilizaremos la antigua denominación ya que al momento de los relatados cambios institucionales llevaba ese nombre.

7. Datos obtenidos del Instituto Nacional de Estadística y Censos de la República Argentina.

8. Art. 5, Ley 11.452, Código Procesal del Menor de la provincia de Santa Fe.

9. De todos modos, ésta sería una hipótesis provisional que requeriría una indagación más profunda.

10. Derogado en 2005 por la ley 11.452, Código Procesal del Menor de la provincia de Santa Fe.

11. Éstos se presentan para retirar a los menores de un lugar de detención.

12. "Art. $1^{\circ}$. No es punible el menor que no haya cumplido 16 años de edad. Tampoco lo es el que no haya cumplido 18 años, respecto de delitos de acción privada o reprimidos con una pena privativa de la libertad que no exceda de dos años, con multa o inhabilitación - Art. $2^{\circ}$ Es punible el menor de dieciséis a dieciocho años de edad que incurriere en delito que no fuera de los enunciados en el art. $1^{\text {" }}$ - Ley 22.278, Régimen Penal de la Minoridad.

13. Art.164 del Código Penal de la Nación Argentina.

14. Establecimos como comienzo del período el año 2001 por ser el momento en que comienza a funcionar la DPMCLP en la provincia de Santa Fe.

15. Se denomina "gatillo fácil" a las ejecuciones llevadas a cabo por miembros de la fuerzas de seguridad en "supuestos" enfrentamientos armados con delincuentes, cuya veracidad es difícil de establecer.

16. Asociación Profesional Policial de la provincia de Santa Fe.

17. La ONG Periodismo Social realiza monitoreos de los principales diarios del país (entre los cuales incluye La Capital, de Rosario) sobre diversos temas (entre ellos niñez y adolescencia), estableciendo la ocurrencia de los mismos en los medios gráficos y analizando qué temática tiene mayor aparición, fuentes consultadas, etc. Para mayor información consúltese: http://www.periodismosocial.org. ar/quienes.cfm?hd=2.

18. Este interrogante excede ampliamente los objetivos del presente artículo. Sin embargo, nos interesa dejarlo planteado, ya que se requiere una reflexión profunda sobre la vinculación entre academia y sociedad, en el sentido de considerar si un medio de llegada tan amplio como es la prensa no permitiría una interacción más fluida entre las producciones de los cientistas sociales y el conjunto de la población. Al respecto puede consultarse J. GLEDHILL (2000), "Antropología y política: compromiso, responsabilidad y ámbito académico", en El poder y sus disfraces. Perspectivas antropológicas de la política, Barcelona, Edicions Bellaterra.

19. Como indicáramos ut supra las estadísticas sobre delitos que están publicadas en la página oficial de Poder Judicial de la provincia de Santa Fe (http://www.poderjudicial-sfe.gov.ar/) contienen la sumatoria de causas de mayores y menores de edad dentro del fuero penal; en consecuencia, podríamos suponer que los datos que maneja el medio gráfico corresponden a ambos grupos de edad.

20. A fines de marzo de 2004 se produce la muerte de un joven luego de su secuestro extorsivo, y su padre, un empresario de la Capital Federal comienza una "cruzada" contra el delito y la inseguridad, exigiendo - a través de manifestaciones que congregan un gran número de personas- el endurecimiento de las penas, la baja de la edad de imputabilidad, etc. Para ampliar este tema consúltese: M. CALZADO (2006), "Elementos para el análisis del tratamiento mediático del caso Blumberg”, Documento de Trabajo No 5, Programa de Antropología Social y Política, Buenos Aires, FLACSO.

21. Dado que nuestro tema de análisis es menores en conflicto con la ley penal, no tomamos los casos 
de menores víctimas de violencia, pero sí nos interesa poder cotejar estos datos, ya que consideramos, nos aportan una visión más relacional del problema que nos permite un abordaje holístico del mismo.

22. Reglas Mínimas de las Naciones Unidas para la Administración de la Justicia de Menores — Reglas de Beijing - ; Directrices de las Naciones Unidas para la Administración de la Justicia Juvenil —Directrices de Ryadh - ; Reglas Mínimas de las Naciones Unidas para los Jóvenes Privados de Libertad, Convención Internacional de los Derechos del Niño.

\section{Bibliografía}

M. BELOFF et al. (2000), Derecho, infancia y familia, Barcelona, Editorial Gedisa.

M. CALZADO (2006), "Elementos para el análisis del tratamiento mediático del caso Blumberg", Documento de Trabajo No 5, Programa de Antropología Social y Política, Buenos Aires, FLACSO.

(2004), "Discursos sociales y prácticas punitivas. Las construcciones significantes de la opinión pública en las asambleas del Plan de Prevención del Delito", en DELITO y Sociedad, Revista de Ciencias Sociales, Año 13, No 20, Buenos Aires, Santa Fe, Universidad Nacional del Litoral.

J. DEGANO (2004), Minoridad y Rehabilitación. Estudio crítico de las prácticas judiciales actuales, Tesis de doctorado, Facultad de Psicología, Rosario, Universidad Nacional de Rosario.

Dirección Nacional de Política Criminal - Ministerio de Justicia y Derechos Humanos de la Nación (2002), Adolescentes infractores a la ley penal, Tribunales Orales de Menores, Informe de la http:// www.polcrim.jus.gov.ar

J. DONZELOT (1990), La policía de las familias, Valencia, Pre-textos.

A. DUBANIEWICZ (1997), Abandono de Menores. Historia y problemática de las instituciones de protección, Buenos Aires, Edigraf.

L. ELBERT; V. VASILE; F. REYES (2002), "Situación normativa de las personas menores de 18 años imputadas de la comisión de un delito", Dirección Nacional de Política Criminal - Ministerio de Justicia y Derechos Humanos de la Nación, en http://www.polcrim.jus.gov.ar

(2002), Violencia Juvenil y Seguridad ciudadana. Problemas y so-

luciones. Informe de la Dirección Nacional de Política Criminal - Ministerio de Justicia y Derechos Humanos de la Nación. http://www.polcrim.jus.gov.ar

E. GARCIA MENDEZ (2004), Infancia. De los derechos y de la justicia, Ciudad Autónoma de Buenos Aires, Editores del Puerto.

et al. (2004), Infancia y democracia en la Argentina. La cuestión de la responsabilidad penal de los adolescentes, Ciudad Autónoma de Buenos Aires, Editores del Puerto.

J. GRINBERG (2004), Características y funcionamiento del entramado burocrático de protección y atención de la infancia en la Ciudad de Buenos Aires. Zonas grises en torno a las intervenciones con chicos, Tesis de Licenciatura en Antropología, Buenos Aires.

S. GUEMUREMAN (2004), "Sistema de producción de información para el monitoreo de adolescentes y jóvenes infractores a la ley penal en Argentina", Documento de Trabajo UNICEF - Oficina de Argentina, en http://www.catedras.fsoc.uba.ar/guemure/bibliografia/Anexo1_IF\%20SG.htm

S. GUEMUREMAN y A. DAROQUI (2001), La niñez ajusticiada, Buenos Aires, Editores del Puerto.

P. HOEL (1979), “La naturaleza de los métodos estadísticos”, en Estadística Elemental, México, Editorial Continental.

G. KESSLER (2006), Sociología del delito amateur, Buenos Aires, Paidós.

L. LARRANDART (1990), "Informe del Grupo de Investigación de Argentina", en Infancia, adolescencia y control social en América Latina. Primer Informe, San José de Costa Rica, 21 a 25 de agosto de 1989, UNICRI-ILANUD, Buenos Aires, Editorial Depalma.

S. MANGUÍA (2004), "Los medios de comunicación y el delito de ser menor", Taller de Infancia - II Congreso Nacional de Sociología, UBA, 20 al 23 de octubre.

S. MARTINI et al. (2002), "Agendas policiales de los medios en la Argentina: la exclusión como un hecho natural”, en Violencias, Delitos y Justicias en la Argentina, Buenos Aires Ed. Manantial.

D. MIGUEZ; A. GONZALEZ (2003), "El Estado como palimpsesto. Control Social, Anomia y Parti- 
cularismo en el sistema penal de menores de la provincia de Buenos Aires, una aproximación etnográfica”, en A. ISLA y D. MIGUEZ (Comp.), Heridas Urbanas. Violencia Delictiva y Transformaciones sociales en los noventa, Buenos Aires, Editorial de las Ciencias, FLACSO.

C. NORIEGA (2002), Nuevos discursos y prácticas en torno a la resolución de conflictos en las Defensorías de Niños, Niñas y Adolescentes del Gobierno de la Ciudad de Buenos Aires, Tesis de Licenciatura en Antropología, Buenos Aires.

Periodismo Social, Niñez y adolescencia en al prensa argentina, Informe Anual, Monitoreo 2004 en www.observatoriojovenes.com.arpublicacionesperiodismoSocialprensa.pdf.

Informe Anual, Monitoreo 2006, en

http://www.capituloinfancia.periodismosocial.net/PS/images/PDF\%20-\%20Final.pdf

D. POLOLA (2004), De Niños y de Menores. Los profesionales y el complejo mapa institucional de la "minoridad" en la ciudad de Rosario, Tesis de licenciatura, Escuela de Antropología, Facultad de Humanidades y Artes, Universidad Nacional del Rosario.

C. RAO (1994), "Principios y estrategias del análisis de datos: Examen cruzado de Datos", en Estadística y verdad. Aprovechamiento del azar, Barcelona, PPU.

C. VARELA (2005), Las representaciones sociales sobre el delito y la "crisis de la seguridad" desde una perspectiva de género, VI Reunión de Antropología del MERCOSUR: Identidad, fragmentación y diversidad, Montevideo, Uruguay, Facultad de Humanidades y Ciencias de la Educación, 16 al 18 de noviembre de 2005.

I. VASILACHIS DE GIALDINO (2003), Pobres, pobreza, identidad y representaciones sociales, Buenos Aires, Gedisa.

C. VILLALTA (1999), JUSTICIA Y MENORES. Taxonomías, metáforas y prácticas, Tesis de Licenciatura en Antropología, Buenos Aires.

\section{Documentos}

Código Penal de la Nación Argentina

Convención sobre los Derechos del Niño - Ley 23.849

Ley 11.452, Código Procesal de Menores de la Provincia de Santa Fe

Ley 26.061, Protección Integral de los Derechos de Niñas, Niños y Adolescentes

Registro $N^{\circ} 1$, Entrevista a Juez de Cámara en lo Penal, octubre de 2007.

Registro No 2, Entrevista a Juez de Menores, diciembre de 2007.

Recibido: 28/02/08. Aceptado: 22/05 